\title{
Utilizing Local and Global Queueing Resources with Uncertainty in State and Service
}

\author{
Edward A. Billard \\ Faculty of Computer Science \& Eng. \\ University of Aizu \\ Fukushima 965-80 Japan
}

\begin{abstract}
In Automonous Decentralized Systems (ADS), there is a high degree of uncertainty, especially in global state information and the performance and reliability of various resources. We model a distributed computer system of local and shared global servers, each of which can process jobs. The local servers insure a degree of autonomy and the global servers provide added computational power and redundancy. When a job arrives, an agent decides whether to schedule the job locally, or whether to ship it to a global server. The agents have access to state information concerning current loads on the global servers and the response times of completed jobs, but because of decentralization this information is delayed, and perhaps lost, along communication channels. The agents must make good decisions under the constraint that service rates are unknown and dynamic. We evaluate a deterministic algorithm and two randomizing algorithms and show that the degree of uncertainty determines which one is the most successful.
\end{abstract}

\section{Introduction}

There are varied instances of Autonomous Decentralized Systems (ADS) and, accordingly, different views of the definition and characteristics of such systems $[1,2,3,4,5]$. We are concerned with the design of computer systems which qualify as ADS, particularly systems with a high degree of physical and logical decentralization $[3,6]$. In the physical dimension, we assume that communication latencies are high relative to local computation times, where the computation is typically some control function. In the logical dimension, we assume a high degree of multilateral decision making, as many agents participate in the control of the system (as described in [7]).

An Autonomous Decentralized System tends to operate in challenging environmental conditions where agents or subsystems must have the ability to perform operations without the direction or assistance of

\author{
Joseph C. Pasquale \\ Department of Computer Science \& Eng. \\ University of California, San Diego \\ La Jolla CA 92093-0114 USA
}

other agents or subsystems. At this level, each agent is self-governing or self-regulating and we call this local autonomy. At higher levels, possibly system-wide, the agents participate in coordination activities and the goal is to make harmonious decisions while still maintaining local autonomy. In this sense, the agents are in an autonomous, or self-governing, body, where cooperative group behavior is important. A decision by an agent may be beneficial to its domain but detrimental to the larger system and vice versa. We believe this balance between local autonomy and cooperative group behavior is an important characteristic of ADS.

There is a high degree of uncertainty in these systems due to the evolving environment and even the evolving subsystems. The uncertainty is compounded by the failure, perhaps frequently, of various subsystems, including communication. These systems appear in mission-critical situations or, at least, in situations where performance and redundancy are important. However, realtime requirements are not as simple as the conventional "hard" and "soft" rules [3].

We model a distributed system that represents an ADS where autonomy, redundancy, and performance are important. The system consists of local and shared global servers, each of which can process jobs. The local servers insure a degree of autonomy and the global servers provide added computational power and redundancy.

Each local server is associated with an independent job arrival stream and a local job-scheduling agent. When a job arrives, the agent decides whether to schedule the job locally, or whether to ship it to a global server. In either case, the job may need to be queued, at the local or global server, as it waits for the processor. Global servers can only receive requests to execute jobs from (remote) agents; they do not have job-scheduling agents, and so all jobs received are directly executed.

The agents must balance the use of the local servers and of the global servers. Although a local server is 
completely under the control of its local agent, there must be cooperative group behavior with respect to the global servers.

The agents have access to state information concerning loads on the global servers and the response times of completed jobs, but this information is delayed, and perhaps lost, along communication channels. The agents must make good decisions under the constraint that the service rate is unknown and $d y-$ namic. These aspects of the model illustrate the challenging environments of ADS. We evaluate a deterministic algorithm and two randomizing algorithms. We show that the degree of uncertainty determines which one is the most successful.

This paper is organized as follows: Section 2 describes other related work; the formal model is presented in Section 3; the algorithms are presented in Section 4 and the performance is evaluated in Section 5. Our conclusions are presented in Section 6.

\section{Other related work}

Autonomous Decentralized Systems [1, 2, 8] can be characterized by on-line expansion, fault-tolerance and on-line maintenance. The environments of these systems are highly dynamic and the systems must continue to operate, in some fashion, under faulty conditions and a high degree of uncertainty. Although not specifically labeled ADS, chaotic systems and computational ecosystems are closely related $[9,10,11]$. In [12], the model of ecosystems is extended to include queueing resources.

Our interests are in the shared use of resources, particularly servers (either computation or factory job), and the effects of uncertainty on the performance. We have previously examined the effects of delayed communication and dynamic group formation $[13,5]$. In ADS, it is important to avoid mutually conflicting decisions [4] and one approach is to use the feedback mechanism of learning automata $[14,15]$. Studies with dynamic job queues $[16,17,18,19,20]$ assume communication, and aged information [21], but assume certainty in service rate. We compare our results to basic queueing analysis for $M / M / c$ systems $[22,23]$ where there are fixed, and known, parameters.

\section{The model}

A model of an ADS in Fig. 1 shows $r$ local agents, each associated with a $\lambda / r$ arrival rate of jobs. The autonomy of each agent is insured by access to a local server with a service rate $\mu_{l}$ and by the restriction that an agent may not access another agent's local server. To increase computational power and redundancy, $R$ global servers with service rate $\mu_{g}$ are also available for the shared use of the local agents. The arrival

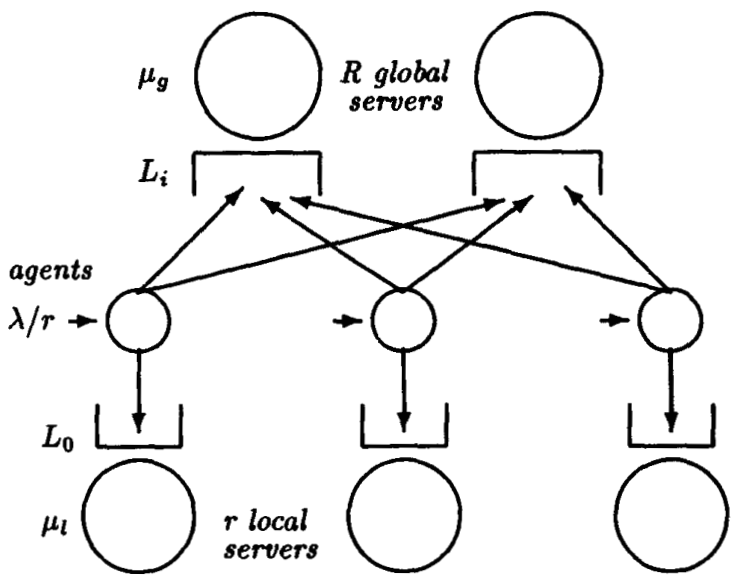

Fig. 1: Agents with Local and Global Servers

and service rates are assumed to be exponentially distributed. Upon the arrival of a job at a local site, a local agent makes decision $D$ concerning the service destination for the job: the local server $(D=0)$ or a global server $(D \in[1, R])$ ?

The agents receive messages, delayed by $d$ seconds, concerning the loads on the global servers (i.e. the lengths of the job queues $L_{i}$ ). This information is distributed to all local agents whenever a job arrives at, or exits from, a global service site. However, there may be periods of time when the information is lost. (The load $L_{0}$ on an agent's local server is known with certainty.) For simplicity, we do not model the actual transmission time of a job (which may or may not be the same as the communication delays), as our main interest is in understanding how communication delay affects information quality. In any particular application, we assume the agents discount the use of global service by the appropriate job transmission time.

Initially, we study environments where the global service rate $\mu_{g}$ is known and static. Subsequently, this assumption is eliminated, and the agents must approximate the dynamic service rate based on communication of the response time $w_{i}$ of each job as it exits from global service site $i$. Also, we consider systems without load information and with only an estimate of the service rate. The goal is to optimize the average response time of all jobs; the challenge is to make good decisions despite aged or lost information and the unknown and dynamic service rates. We note that other metrics may be substituted here, but agents will tend to face similar obstacles due to uncertainty. 


\section{Algorithms}

We present three algorithms with the goal to investigate the success of these algorithms in environments with varying degrees of uncertainty.

From standard queueing theory, the expected response time of a new job queued at server $i \in[0, R]$ is:

$$
w_{i}=\left\{\begin{array}{ll}
\left(L_{i}+1\right) / \mu_{l} & \text { if } i=0 \\
\left(L_{i}+1\right) / \mu_{g} & \text { otherwise }
\end{array} .\right.
$$

The first algorithm is a simple deterministic algorithm: select the server which has the minimum expected response time; that is,

$$
\text { Alg. DET: } D=\min _{i} w_{i} \text {. }
$$

(If there are multiple optima, then the algorithm randomizes over the optima.) This algorithm is quite obvious and certainly used in many systems as it is a heterogeneous version of "send the job to the shortest queue". We shall show that this algorithm works well in environments with a high degree of certainty.

The second algorithm, Time-Space Randomization, is based upon a randomizing technique for coordination without communication [4], and was extended in another study [24] to distinguish between local and global servers. The algorithm assumes that no state information is available. This can be due to numerous reasons: whatever information may be available is so poor it is worth ignoring; the inability to communicate state information is a permanent feature of the environment; the state-information communication system has failed. In any case, the algorithm randomizes to balance the load offered to each server in relation to the service rates and is independent of the actual load situation.

Upon the arrival of a job, the probability that the agent will offload the job to a global server is called the access probability $\alpha$. This variable determines the fraction of jobs that will be offloaded for global service, and randomizes over the time dimension. If the agent decides to offload the job, the agent randomizes again over the space dimension of global servers; that is, each global server is selected with probability $1 / R$. For the case $\mu_{l}=\mu_{g}$, the optimal access probability is

$$
\alpha^{*}=\frac{R}{r+R},
$$

that is, the load is distributed to the global servers in the same proportion as the number of global servers to total servers (but the general case is more complicated, involving the arrival rate $\lambda$ [24]). If $r=R=10$, $50 \%$ of the jobs should be kept local and the other
$50 \%$ should be evenly distributed among the 10 global servers. Each local and global site is offered $\lambda / 20$ jobs per sec. In summary, the probability that server $i$ will be selected is:

$$
\text { Alg. } T S: p_{i}=\operatorname{Pr}[D=i]=\left\{\begin{array}{ll}
1-\alpha^{*} & \text { if } i=0 \\
\alpha^{*} / R & \text { otherwise }
\end{array}\right. \text {. }
$$

The algorithm ignores current load information (we call this a static randomizing algorithm); however, it is expected to perform as best as can be expected in environments with total uncertainty.

The third and last algorithm has similarities to both of the preceding algorithms. The algorithm favors the selection of servers with shorter job queues, and does so with non-uniform randomization. If $\mu_{l}=\mu_{g}$ and the job queues happen to be balanced (which is always the goal), then the algorithm defaults to Time-Space Randomization (which is a balancing algorithm). Formally, the weighted randomizing algorithm selects server $i$ with probability:

$$
\text { Alg. } W R: p_{i}=\operatorname{Pr}[D=i]=\left[W_{i} \sum_{j=0}^{R} \frac{1}{W_{j}}\right]^{-1}
$$

where

$$
W_{i}=\left\{\begin{array}{ll}
w_{i} & \text { if } i=0 \\
w_{i} r & \text { otherwise }
\end{array} .\right.
$$

Let us examine the algorithm in more detail but, first, let us ignore the important factor of $r$ (i.e. the number of local servers) in the computation of $W_{i}$ in (2). The probability $p_{i}$ is based on the expected response time $w_{i}$ in relation to all of the response times $w_{j}$. That is, if $w_{i}$ is half the value of a particular $w_{j}$, then $i$ should be selected with twice the probability of $j$. If $w_{i}=w_{j}$, then both should be selected with equal probability. Besides this property of proportionality, the algorithm guarantees $\sum p_{i}=1$.

Now let us consider the factor of $r$ in the computation of $W_{i}$ in (2). If all of the expected response times are identical, an important question arises: should the probability of selecting the local server be the same as any of the global servers $\left(p_{i}=1 /(R+1)\right)$ ? If $r>1$, the answer is "no". All of the local servers use the same algorithm and participate in the state evolution of the global servers. As stated earlier, a local agent must balance the need for global service against the needs of other agents. The agents must cooperate and randomize appropriately. In the Time-Space Randomization example, the agent should keep $50 \%$ of the load $\left(p_{0}=.5\right)$ and a global server should be selected with probability $p_{i}=.05$. Of course, if there is only a single local agent, then the load should be assigned with equal probability. 


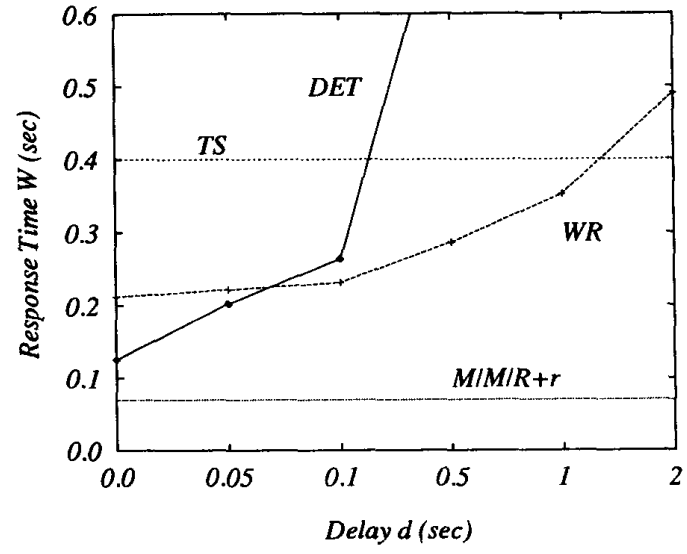

Fig. 2: Delays in Load Information

It is the factor of $r$ in (2) that yields this cooperative behavior and, alternatively, represents the proportion of the service rate $\mu_{g}$ that a local agent "owns" as a member of the $r$ local agents.

If the queues are balanced, the global server access probability for the $W R$ algorithm is

$$
\alpha=\frac{R \mu_{g}}{r \mu_{l}+R \mu_{g}}
$$

and, if $\mu_{l}=\mu_{g}$, this agrees with $\alpha^{*}$ (1) for the TS algorithm. Note that (3) attempts to maintain balance according to proportional service rates but does not accommodate the influence of the arrival rate at each server on the overall response time [24].

\section{Experiments}

In this section, we evaluate the three algorithms in environments with aged information and with uncertainty in the service rates. Aged information results from either fixed delays in message transmission, a regular fractional loss of messages, or a short episode where all messages are lost. Uncertainty in service rates results from agents accumulating the most recent response times of jobs or by a faulty fixed estimate of the rate. The goal is to show that the best algorithm depends on the nature and extent of the uncertainty.

\subsection{Communication delays}

Fig. 2 shows the results with different delays in communication (note the non-linear scale) and each point is the average of 5 runs over 1000 seconds. All the results in this study assume that $\lambda=150, R=r=10$ and $\mu_{g}=\mu_{l}=10$ (at least for the static global service experiments). These values have been chosen such that the agents can achieve a steady-state solution only by the shared use of the global servers.

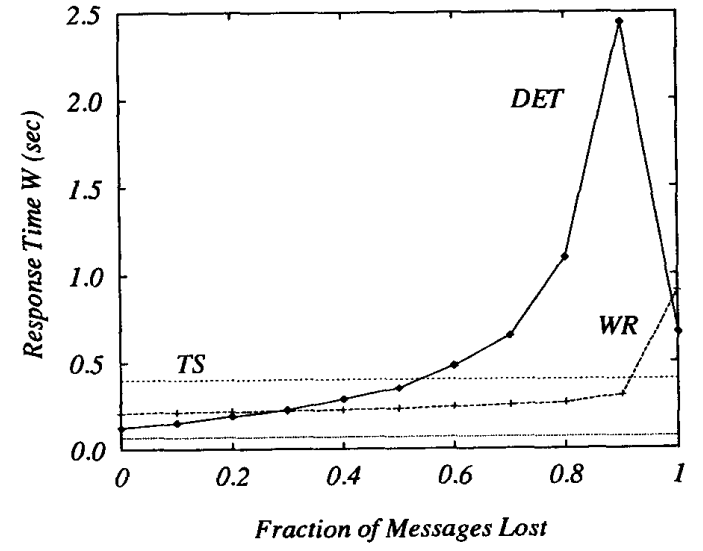

Fig. 3: Effects of Fractional Loss of Messages

In Fig. 2, the $D E T$ and $W R$ algorithms apply decision making to an aged view of the load $L_{i}$ of the global servers. As a benchmark, we show the performance of a system with a centralized controller and perfect information regarding all $R+r$ servers. This is a $M / M / R+r$ queueing system and is not considered a realistic model for an Autonomous Decentralized System. With certainty of information $(d=0)$, the loss of the $D E T$ algorithm compared to the centralized case is due to the autonomy of the local agents; that is, the local servers are not shared and jobs cannot be offloaded among them (each local server is an $M / M / 1$ queueing system).

The performance of the TS algorithm is determined analytically [24] and is independent of the delays in the system as it ignores current load conditions. The results show that the $D E T$ algorithm is the most successful of the three with highly certain information ( $d \leq .05$ seconds). However, the $W R$ algorithm is the most successful for a broad range of delays. The TS algorithm has the best performance given the highest delays as the out-of-date load information actually hinders good decision making. With large delays in the communication system, an apparently lightlyloaded server can become the target of too many offloading agents and oscillatory and chaotic behavior have been observed $[9,10]$. These sensitivities must be considered in the design of systems which operate under high degrees of uncertainty.

\subsection{Communication failure}

We examine two types of communication failure, both of which affect the age of information. The first type, in Fig. 3, shows the performance as a function of the fraction of broadcast messages lost over the run of the experiment, with successful messages delivered in- 


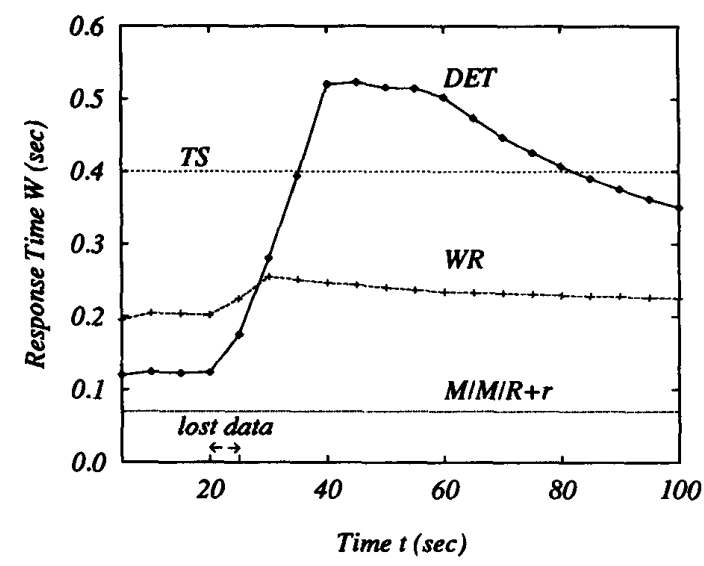

Fig. 4: Effects of Lost Load Information

stantaneously. With a small loss (i.e. the information is relatively certain), the $D E T$ algorithm has the best performance. Over a broader range of uncertainty, i.e. fraction of lost messages, the $W R$ algorithm is best and the $D E T$ algorithm suffers a substantial loss in performance. If all messages are lost, the $T S$ algorithm actually outperforms the other two. Note that in this case, the load information is still the initialized value $\left(L_{i}=0\right)$ but the local service load $\left(L_{0}\right)$ is known with certainty. The $D E T$ and $W R$ algorithms both have a bias to keep jobs local when ties occur between the local and global servers. When all messages are lost, the performance of the $D E T$ algorithm actually improves, as compared to the case when $90 \%$ of the messages are lost. The data is very misleading when only $10 \%$ of the messages arrive and the algorithm over-reacts by sending too many jobs to the apparently lightly-loaded servers.

The second type of failure, in Fig. 4, shows the performance of the algorithms during an episode of communication failure. (The data is the average of 5 runs over the 100 second interval and the response time $W(t)$ is the average up to time $t$ ). All messages concerning load information are lost for $20 \leq t \leq 25$; otherwise, the messages are delivered instantaneously. Again, the TS algorithm is unaffected by load information but the $D E T$ algorithm suffers a substantial loss as it relies heavily on exact loads. Many jobs are sent to the least loaded server at the time of communication failure and, even by the end of the experiment, the system has not fully recovered. The $W R$ algorithm has a small loss as it continues to use the same probabilities at the time of communication failure, which approximate a balanced system. This is one of the strongest arguments for using a randomizing algorithm.

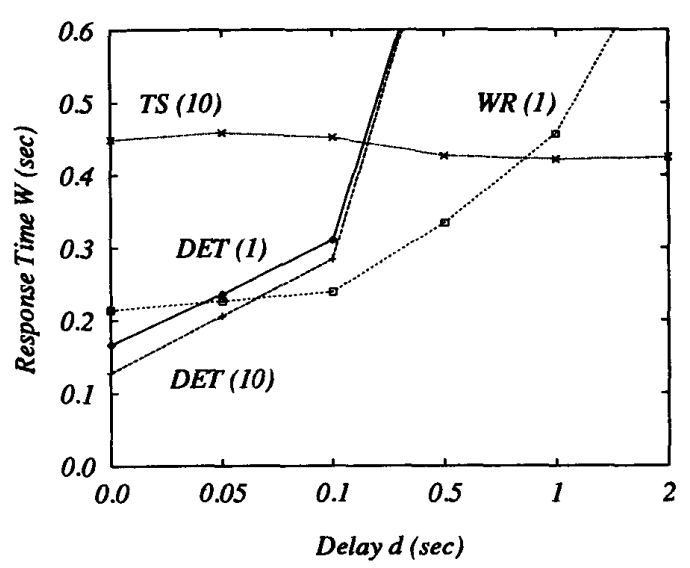

Fig. 5: Accumulating Response Times

\subsection{Accumulating response time}

We increase the level of uncertainty by considering systems where the global service rate $\mu_{g}$ is not a parameter given to the decision-making agents. Instead, the agents approximate the effective service rate from the individual response times of jobs. As a job is completed by global server $i$, a message is broadcast with the response time $x_{i}$ of the job along with the current queue length $L_{i}$. Again, this information may be delayed or lost.

The agents accumulate this information and we examine the three algorithms with respect to different moving averages. For example, $D E T(10)$ is the deterministic algorithm with the expected global service response time $w_{i}=L_{i} \bar{x}_{i}$, where $\bar{x}_{i}$ is the average of the last 10 observations from server $i$. The $W R(10)$ algorithm is similar but the $T S(10)$ algorithm computes a general estimate for global service:

$$
\mu_{g}=(1 / R) \sum_{i=1}^{R} \bar{L}_{i} / \bar{x}_{i},
$$

where $\bar{L}_{i}$ is the average of the last 10 observations. In this case, it is assumed that the $T S$ algorithm has access to information, albeit delayed.

Fig. 5 shows the performance of the system with different delays in the messages and with constant service rate $\left(\mu_{g}=10\right.$, as before, but this is not known by the agents). The $T S$ algorithm performs slightly worse than the predicted value from the previous experiments where $\mu_{g}$ is given and requires at least 10 points to achieve this level. The performance of the $W R$ algorithm was found to be independent of the number of values in the moving average and, as such, only $W R(1)$ is shown. The randomization mechanism 


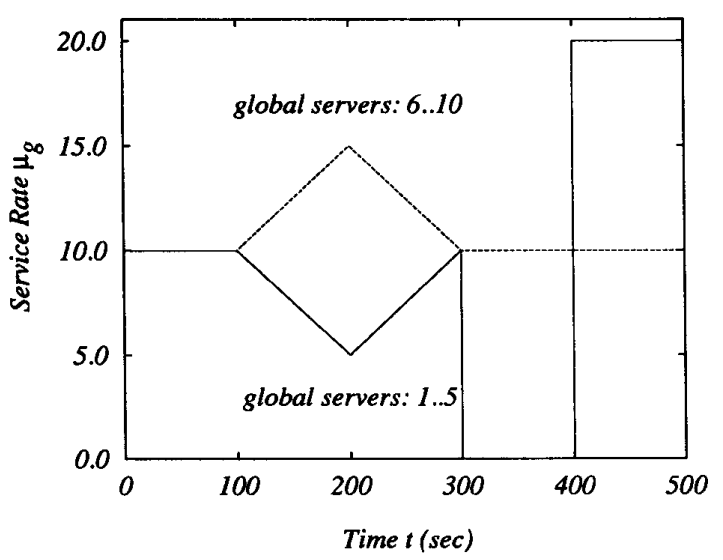

Fig. 6: Dynamic Service Rate

is effective with just the most recent response time from each global server. The $D E T$ algorithm shows some change with the number of observations as it is more sensitive to the expected response times.

The agents are able to detect the effective service rates and we draw the same conclusion concerning Fig. 2: the most successful algorithm depends on the uncertainty of information. Again, the $W R$ algorithm is the best algorithm over a broad range of delays but not at either extreme.

In Fig. 6, we hypothesize an episode of dynamic global service (i.e. $\left.\mu_{g}(t)\right)$ and, at any instant, the rate is assumed to be exponentially distributed. This episode is a stylized version of more arbitrary behavior that can be found in Autonomous Decentralized Systems but suffices to demonstrate our points. After a static period, the service rates of half of the global servers tend to increase as the service rates of the other half decrease. This is followed by a period of readjustment. Next, half of the servers are taken off-line (or refuse service) for a substantial period (here, the redundancy is important) and then come on-line with improved service. This episode illustrates both dynamic service rates and the on-line nature of many ADS.

In Fig. 7, we examine the performance of the algorithms during this episode, with communication assumed to be instantaneous. The $T S$ algorithm performs poorly as it uses a single estimate of the global service rate and cannot accommodate individual rates. The performance of the $D E T$ and $W R$ algorithms is similar except late in the episode when the $D E T$ algorithm is more responsive to the new high-powered servers. In this instance, the sensitivity of the algorithm is to its advantage, but in the earlier experi-

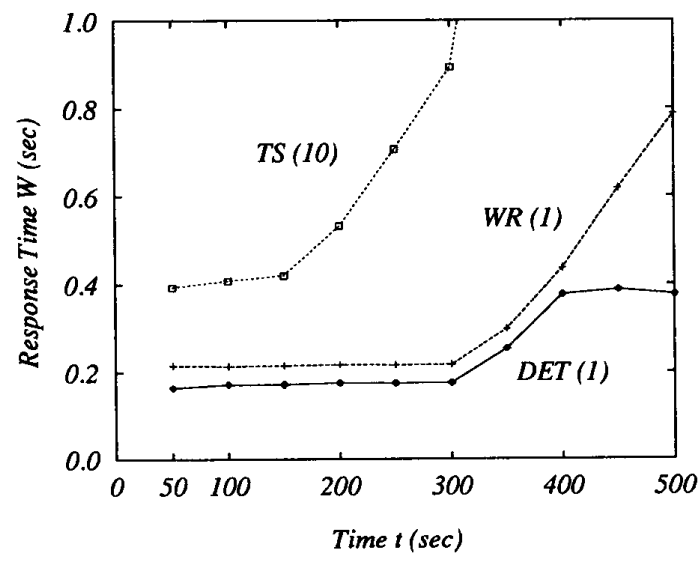

Fig. 7: Dynamic Service without Delays

ments, the sensitivity was a distinct disadvantage.

In Fig. 8, we consider the performance over the entire episode of dynamic service for different delays in communication. With low uncertainty $(d \leq .1)$, the $D E T$ algorithm is the most successful as expected from previous experiments. The $W R$ algorithm is the best over a range of delays, some of which is not shown, and the $T S$ algorithm is very poor unless substantial delays are introduced.

\subsection{Estimating global service}

In this section, we consider systems without load information and agents which have deduced, or have been provided, a fixed estimate $\mu_{g}$ of the global service rate (which tends to be accepted as an input parameter in most queueing studies). The results of our analysis of the TS algorithm shows the effects of under- or over-estimating the true value $\mu$.

The agents make their decisions, as before, as to the optimal access probability based on $\mu_{g}$. Let $\alpha^{*}(\mu)$ be the optimal access probability for a particular $\mu$. We define a new gain function,

$$
G=\frac{W\left(\alpha^{*}\left(\mu_{g}\right)\right)}{W\left(\alpha^{*}(\mu)\right)}
$$

which is the fraction of the actual response time resulting from the service estimate compared to the response time that could have been achieved if the actual service rate were known.

This gain function is plotted in Fig. 9 as a function of the actual service rate $\mu$. In this example, the estimated service rate $\mu_{g}=10$ and the gain function is one for $\mu=10$. On either side of this value, there is inaccuracy in the estimated service rate and an associated loss. If the service rate is under-estimated $\left(\mu>\mu_{g}\right)$, 


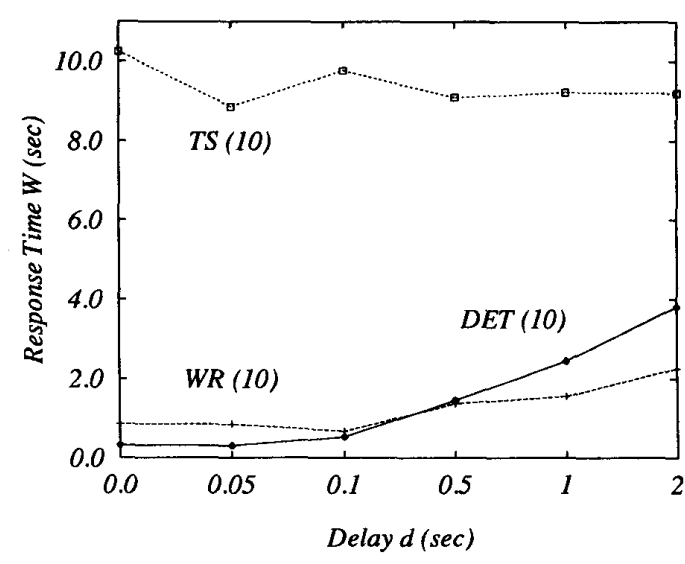

Fig. 8: Effects of Dynamic Service Rate

there is a loss due to not accessing the global servers sufficiently and this loss is exacerbated with increasing numbers of global servers. If the service rate is over-estimated $\left(\mu<\mu_{g}\right)$, then the global servers are accessed too frequently.

If the inaccuracy is sufficient (in over-estimation), the response time becomes worse than $r$ isolated $M / M / 1$ subsystems which ignore the global servers. This occurs, in this example, at $\mu \approx 7$ for all four values of $R$. This isolation threshold can be determined analytically [24] and, obviously, if the agents know the actual service rate is below this threshold, they can either adjust the access probability or avoid the global servers entirely. It is precisely the point that the agents do not know the actual service rate that has created the obstacle. Realistically, if a probability distribution for the service is available, the agents may select the optimal access probability based on a weighted response time function.

\section{Conclusions}

The model presented in this study captures autonomy as well as the problem of accessing global servers under various degrees of uncertainty. In particular, the two randomizing algorithms $T S$ and $W R$ are intended to cooperatively share the global servers. The uncertainty is due to aged information (delayed or lost messages) and a dynamic or unknown service rate. The deterministic algorithm DET operates best with low uncertainty, the weighted randomizing algorithm WR operates best over a broad range of delays in dynamic environments, and the static uniformly randomizing algorithm $T S$ is suited for systems where the load information is unavailable or so old as to be a detriment to decision making.

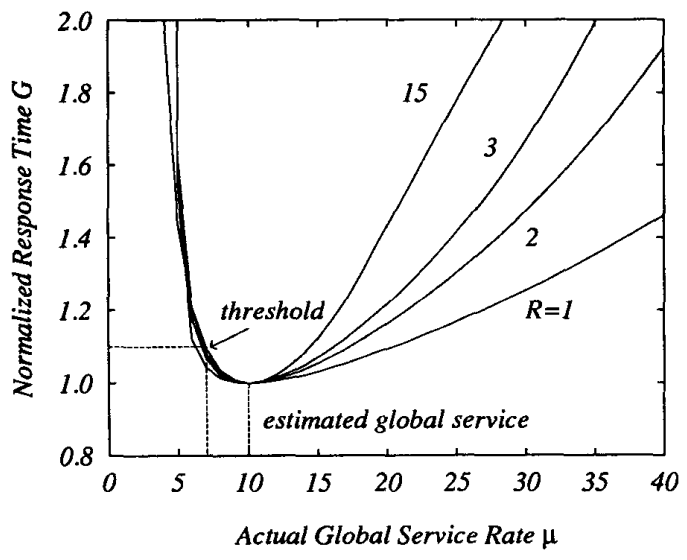

Fig. 9: Loss Due to Estimation Error

The weighted randomizing algorithm should be considered as the best choice overall in many systems. However, a combination of the three algorithms can be attempted if information is available regarding the appropriate instances to switch mechanisms. For example, the weighted randomizing algorithm can be substituted dynamically for the deterministic algorithm if the age of information exceeds a threshold (and similarly for the static uniformly randomizing algorithm). However, the determination of the proper algorithm is based on observational data which may be different for different agents. That is, all three algorithms may be applied at the same time by different populations of agents. This tests the cooperative behavior as the randomizing algorithms assume similar behavior among the agents.

\section{References}

[1] K. Mori, "Autonomous decentralized systems: Concept, data field architecture and future trends," in Proc. IEEE Intl. Symp. Autonomous Decentralized Syst., pp. 28-34, Mar. 1993.

[2] H. Ihara and K. Mori, "Autonomous decentralized computer control systems," IEEE Computer, vol. 17, pp. 57-66, Aug. 1984.

[3] E. Jensen, "A timeliness model for asynchronous decentralized computer systems," in Proc. IEEE Intl. Symp. Autonomous Decentralized Syst., pp. 173-182, 1993.

[4] J. Pasquale, "Randomized coordination in an autonomous decentralized system," in Proc. IEEE Intl. Symp. Autonomous Decentralized Syst., pp. 77-82, 1993. 
[5] E. Billard and J. Pasquale, "Dynamic scope of control in decentralized job scheduling," in Proc. IEEE Intl. Symp. Autonomous Decentralized Syst., pp. 183-189, 1993.

[6] E. Jensen, "Decentralized executive control of computers," in Proc. IEEE Intl. Conf. Distr. Comput. Syst., pp. 31-35, 1982.

[7] E. Jensen, "Distributed control," in Distributed Systems - Architecture and Implementation, an Advance Course (B. Lampson, M. Paul, and H. Siegert, eds.), New York: Springer-Verlag, 1981.

[8] D. Ferguson, C. Nikolaou, and Y. Yemini, "An economy for managing replicated data in autonomous decentralized systems," in Proc. IEEE Intl. Symp. Autonomous Decentralized Syst., pp. 367-375, Mar. 1993.

[9] B. A. Huberman and T. Hogg, "The behavior of computational ecologies," in The Ecology of Computation (B. A. Huberman, ed.), North-Holland: Elsevier Science Publishers, 1988.

[10] T. Hogg and B. A. Huberman, "Controlling chaos in distributed systems," IEEE Trans. Syst., Man, Cybern., vol. 21, pp. 1325-1332, Nov./Dec. 1991.

[11] J. O. Kephart, T. Hogg, and B. A. Huberman, "Dynamics of computational ecosystems," Phys. Rev. $A$, vol. 40, pp. 404-421, July 1989.

[12] E. Billard, "Controlling instability in distributed queueing systems," in Proc. IEEE Intl. Symp. Intelligent Control, pp. 111-117, 1994.

[13] E. Billard and J. Pasquale, "Effects of delayed communication in dynamic group formation," IEEE Trans. Syst., Man, Cybern., vol. 23, pp. 1265-1275, Sept./Oct. 1993.

[14] A. Glockner and J. Pasquale, "Coadaptive behavior in a simple distributed job scheduling system," IEEE Trans. Syst., Man, Cybern., vol. 23, pp. 902-907, May/June 1993.
[15] R. Mirchandaney and J. Stankovic, "Using stochastic learning automata for job scheduling in distributed processing systems," J. Parallel and Distr. Comput., vol. 3, pp. 527-552, 1986.

[16] A. Winckler, "A distributed look-ahead algorithm for scheduling interdependent tasks," in Proc. IEEE Intl. Symp. Autonomous Decentralized Syst., pp. 190-197, Mar. 1993.

[17] R. Mirchadaney, D. Towsley, and J. Stankovic, "Analysis of the effects of delays in load sharing," IEEE Trans. Comput., vol. 38, pp. 1513-1525, Nov. 1989.

[18] D. Eager, E. Lazowska, and J. Zahorjan, "Adaptive load sharing in homogeneous distributed systems," IEEE Trans. Software Eng., vol. SE-12, pp. 662-675, 1986.

[19] S. Zhou, "A trace-drive simulation study of dynamic load balancing," IEEE Trans. Software Eng., vol. 14, pp. 1327-1341, Sept. 1988.

[20] K. Shin and Y. Chang, "Load sharing in distributed real-time systems with state-change broadcasts," IEEE Trans. Comput., vol. 38, pp. 1124-1142, Aug. 1989.

[21] T. Casavant and J. Kuhl, "A formal model of distributed decision-making and its application to distributed load balancing," in Proc. IEEE Intl. Conf. Distr. Comput. Syst., pp. 232-239, Sept. 1986.

[22] A. Allen, Probability, Statistics, and Queueing Theory with Computer Science Applications. New York: Academic Press, 1977.

[23] D. Gross and C. Harris, Fundamentals of Queueing Theory. New York: John Wiley \& Sons, 1985.

[24] E. Billard, "Autonomous agents sharing global resources without communication," Tech. Rep. 94-1-005, University of Aizu, 1994. 